\title{
Filament Preserving Model (FPM) Segmentation Applied to SAR Sea-Ice Imagery
}

\author{
Qiyao Yu and David A. Clausi, Senior Member, IEEE
}

\begin{abstract}
Modeling spatial context constraints using a Markov random field (MRF) has been widely used in the segmentation of noisy images. Its applicability to synthetic aperture radar (SAR) sea-ice segmentation has also been demonstrated recently. However, most existing MRF models are not capable of preserving filaments, specifically leads and ridges for SAR sea ice, which are valuable for ship navigation applications and necessary for identifying certain ice types. In this paper, a new statistical context model is proposed that, within the same scene, can simultaneously preserve narrow elongated features while producing similar smooth segmentation results comparable to typical MRF-based approaches. Tested on one synthetic image and two SAR sea-ice scenes, this filament preserving model substantially improves classification accuracies when compared to standard Gaussian mixture and MRF-based segmentation algorithms.
\end{abstract}

Index Terms-Adaptive model, egg code, lead, Markov random field (MRF), ridge, synthetic aperture radar (SAR).

\section{INTRODUCTION}

$\mathbf{S}$ YNTHETIC aperture radar (SAR) has been intensively used in monitoring and understanding of sea ice during the past decade. Important applications of SAR sea ice analysis include both scientific and operational activities such as climate research and ship navigation. The interpretation of SAR sea ice data currently in operation at many ice services [e.g., the Canadian Ice Service (CIS)] is in the form of a manually produced ice chart. In an ice chart, each outlined region corresponds to a visually homogeneous area in the SAR image and is associated with an egg code defined by the World Meteorology Organization [1]. The egg code provides information about the type, concentration, and floe size of the ice components in the region. Such an interpretation is limited in throughput, has human bias, and does not classify at pixel level resolution. Analysis methods that are as fully automated as possible with minimum user interactions are thus desired. As a fundamental step of the analysis process, a robust and accurate computer-assisted image segmentation method must be designed. However, SAR sea ice imagery is extremely complex and highly variable due to numerous environmental factors, imaging parameters, and the notorious speckle noise. As a result, to design an automated segmentation technique sufficiently effective and robust for operational use is very difficult.

Manuscript received April 10, 2006; revised June 19, 2006. This work was supported by the Natural Sciences and Engineering Research Council of Canada (NSERC) Networks of Centres of Excellence (NCE) called Geomatics for Informed Decisions (GEOIDE), Canadian Ice Service (CIS), and Cryospheric System in Canada (CRYSYS).

The authors are with the Department of Systems Design Engineering, University of Waterloo, Waterloo, ON N2L 3G1, Canada (e-mail: q2yu@engmail.uwaterloo.ca; dclausi@engmail.uwaterloo.ca).

Digital Object Identifier 10.1109/TGRS.2006.885046
Previous research [2]-[5] has demonstrated some degree of success using tone (gray level) and texture features in a supervised manner (i.e., class distribution known a priori by training) on images of sea ice of limited types, locations, and seasons. Some more recent papers choose unsupervised segmentation techniques as they can theoretically adapt to the varying property of backscatter characteristics of SAR sea ice imagery more effectively. Soh and Tsatsoulis [6] developed a dynamic thresholding and spatial clustering algorithm applied to the SAR sea ice tone feature. Samadani [7] assumed that the tone of each ice class in SAR imagery follows a Gamma distribution, and used a finite Gamma mixture model for the clustering. Clausi and Yue [8] also adopted a clustering technique but applied it to the texture features. In [9], a complete interpretation system named ARKTOS was designed with the watershed and region growing techniques chosen for the segmentation task. Karvonen [10] developed a system based on a pulse-coupled neural network. Deng and Clausi [11] implemented a Markov random field (MRF) model to account for the spatial relationship among pixels for producing segmentation results less sensitive to the SAR speckle noise.

The MRF-based approaches are attractive, as they allow to take into account the nature of speckle noise in a statistically sound manner and at the same time provide an efficient regularization for the general ill-posed segmentation problem. Moreover, it is very convenient for such global approaches to incorporate user provided information such as the egg code, which could be beneficial to the reliability of the system. Currently, such a semiautomated analysis process seems more robust and hence more practical than fully automated ones.

Therefore, this paper follows the MRF-based approaches, using a statistical model to incorporate a spatial context constraint and aiming at segmenting regions into component ice types. However, this paper differs from others in that it focuses on simultaneously preserving filaments (narrow linear features), such as ridges and leads. Ridges are irregular bright filaments on ice floes corresponding to pressure deformation features, and leads are generally dark filaments corresponding to cracks that ships can navigate through. They are important for applications such as ship routing, the safety of offshore structures, and regional climate models. For example, regional climate models are especially sensitive to total ice concentration estimates due to the climatic difference in thermodynamic fluxes under ice versus no ice conditions in cold regions. Moreover, the existence of ridges or leads is also crucial for identifying certain ice types such as gray and gray-white ice. Existing MRF-based approaches generally discard such filament features since the models are isotropic and cannot describe narrow elongated features. By this motivation, we have designed our model to be adaptive to the existence of filaments and to be able to 
capture filament features without compromising the strength on suppressing noisy configurations in other parts of the image.

This paper is unique compared to direct ridge and lead extraction methods. For example in [12], ridges or leads are extracted by a line detector combined with a thresholding operation. Our goal, however, is the segmentation of different ice types with the filaments preserved at the same time. Although the output of the proposed method is not a map of ridges and leads, derivation of those ridges and leads from the output is straightforward. To the best of the authors' knowledge, such an approach (region consistency and lead preservation in SAR imagery) has not been previously considered.

Similar research also exists in fields other than SAR sea ice image classification. There are some adaptive neighborhood MRF methods [13], [14] in the literature that select the MRF neighbors using external knowledge or by incorporating a local degree of homogeneity. The proposed model in this paper is unique since it is tailored to filaments and does not need any external knowledge.

The organization of this paper is as follows. After a brief review of MRF-based segmentation in Section II, the filament preserving model (FPM) is introduced in Section III. Section IV presents the experiments and discussions. Summary and future work comprise Section V.

\section{Classical MRF-Based Segmentation}

The MRF provides a method to model the joint probability distribution of the image sites in terms of the local spatial interactions. It assumes that each site is independent of other sites outside of its defined neighborhood given the configuration of neighborhood sites. For any configuration $\mathbf{x}$ of the state space $T$ of random field $X$, its joint probability is defined by a Gibbs distribution [15]

$$
P(X=\mathbf{x})=\frac{1}{Z} \exp \{-E(\mathbf{x})\}=\frac{1}{Z} \exp \left\{-\sum_{c \in C} V_{c}(\mathbf{x})\right\}
$$

where $C$ is the set of cliques which are defined as the sets of mutually neighboring sites, $V_{c}(\mathbf{x})$ is the energy of configuration $\mathbf{x}$ on clique $c, E(\mathbf{x})$ is the total energy of configuration $\mathbf{x}$, and $Z$ is the normalizing constant.

The MRF has been popular for image segmentation in modeling spatial context [11], [16]-[18] which, with the segmentation task formulated as a maximum a posterior (MAP) problem under the Bayesian framework in (2), corresponds to the prior $P(\mathbf{x})$ in the equation

$$
\arg \max _{\mathbf{x}} P(\mathbf{x} \mid \mathbf{y})=\arg \max _{\mathbf{x}} p(\mathbf{y} \mid \mathbf{x}) P(\mathbf{x})
$$

Here, $\mathbf{y}$ represents the observations and $\mathbf{x}$ represents the class labels. A typical MRF spatial context model is the multilevel logistic model (MLL) [16]. The MLL has only pair-site cliques with energy defined as

$$
V_{c}\left(x_{s}, x_{t}\right)= \begin{cases}\beta, & \text { if } x_{s} \neq x_{t} \\ 0, & \text { otherwise }\end{cases}
$$

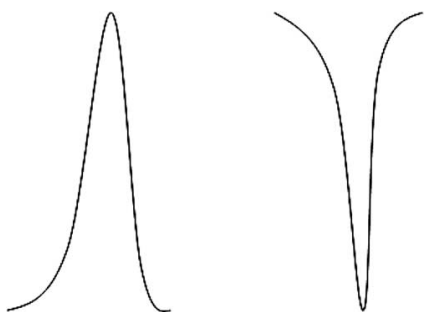

Fig. 1. Shape of ridge and valley in the main principle curvature direction.

where $x_{s}$ and $x_{t}$ are the class labels of the two neighboring sites $s$ and $t$, respectively, and $\beta$ is a positive number. With such a model, the prior $P(\mathbf{x})$ is large if local neighborhood region is dominated by one single class and small otherwise. Although the MLL is efficient in suppressing noisy configurations, it merges narrow elongated segments into surrounding classes due to its deficiency in describing anisotropic behavior.

\section{Filament Preserving Model (FPM)}

The new model is desired to be adaptive to the existence of filaments. Since filaments are narrow and elongated, they are expected to have a sharp ridge or valley shape (intensity as height) in the main principle curvature direction (i.e., the direction that has the shortest crossing path of the filament at the specified site) as depicted in Fig. 1. Therefore, a new feature measured as the curvature of the shape is introduced to provide evidence for the existence of filaments, and is thus named filament strength. Detailed extraction of filament strength is found in Section III-A.

Let $\mathbf{y}^{(f)}$ represents the filament strength vector on the image lattice $S$, and $y_{s}^{(f)}$ the filament strength on site $s \in S$. Similarly, $\mathbf{y}$ and $y_{s}$ denotes, respectively, the gray level on the image lattice $S$ and on site $s \in S$. Following the naive Bayes approach [19], an assumption is made that $y_{s}$ depends only on $x_{s}$, and $y_{s}^{(f)}$ depends only on $x_{s}$ and the neighborhood class labels $\mathbf{x}_{\eta_{s}}$, where $\eta_{s}$ is the neighborhood of site $s$. The MAP segmentation in (2) then becomes

$$
\arg \max _{\mathbf{x}} \prod_{s \in S}\left\{p\left(y_{s} \mid x_{s}\right) p\left(y_{s}^{(f)} \mid x_{s}, \mathbf{x}_{\eta_{s}}\right)\right\} P(\mathbf{x})
$$

Modeling $p\left(y_{s}^{(f)} \mid x_{s}, \mathbf{x}_{\eta_{s}}\right)$ is difficult, as the configuration space of $s$ together with $\eta_{s}$ has a high dimension. We thus introduce a Boolean variable which indicates whether the site belongs to a filament or not, and name it filament flag. Let $x_{s}^{(f)}$ denote the filament flag for site $s$ with $x_{s}^{(f)}=0$ corresponding to nonfilament and $x_{s}^{(f)}=1$ filament, and assume $y_{s}^{(f)}$ to be independently distributed given $x_{s}^{(f)}$. Using the principle of iterated conditional mode [20], the MAP segmentation is approximated by performing iteratively at each lattice site $s$ the following:

$$
\arg \max _{x_{s}} p\left(y_{s} \mid x_{s}\right) \sum_{x_{s}^{(f)}}\left\{p\left(y_{s}^{(f)} \mid x_{s}^{(f)}\right) P\left(x_{s}, x_{s}^{(f)} \mid \hat{\mathbf{x}}_{\eta_{s}}\right)\right\}
$$


where $\hat{\mathbf{x}}_{\eta_{s}}$ is the provisional estimate of the neighborhood configuration $\mathbf{x}_{\eta_{s}}$. The probability $P\left(x_{s}, x_{s}^{(f)} \mid \hat{\mathbf{x}}_{\eta_{s}}\right)$ can be further decomposed as $P\left(x_{s}^{(f)} \mid x_{s}, \hat{\mathbf{x}}_{\eta_{s}}\right) \cdot P\left(x_{s} \mid \hat{\mathbf{x}}_{\eta_{s}}\right)$. To make the model of $P\left(x_{s} \mid \hat{\mathbf{x}}_{\eta_{s}}\right)$ capable of describing anisotropic behaviors with simple pair-site cliques, we include filament flag into it and hence use $P\left(x_{s} \mid x_{s}^{(f)}, \mathbf{x}_{\eta_{s}}^{(f)}, \hat{\mathbf{x}}_{\eta_{s}}\right)$ instead, where $\mathbf{x}_{\eta_{s}}^{(f)}$ is the filament flags of $\eta_{s}$. For representation convenience, $\hat{\mathbf{x}}_{\eta_{s}}$ is denoted by $\mathbf{x}_{\eta_{s}}$, and (5) becomes

$$
\begin{aligned}
\arg \max _{x_{s}} p\left(y_{s} \mid x_{s}\right) \sum_{x_{s}^{(f)}}\left\{p\left(y_{s}^{(f)} \mid x_{s}^{(f)}\right)\right. \\
\left.\quad \times P\left(x_{s}^{(f)} \mid x_{s}, \mathbf{x}_{\eta_{s}}\right) P\left(x_{s} \mid x_{s}^{(f)}, \mathbf{x}_{\eta_{s}}^{(f)}, \mathbf{x}_{\eta_{s}}\right)\right\} .
\end{aligned}
$$

It should be noted that such an approximation of (4) using (6) has caused the class label field to be non-Markov unless $P\left(x_{s}^{(f)} \mid x_{s}, \mathbf{x}_{\eta_{s}}\right)$ is defined to be either 0 or 1 . We can also include the filament flag as the unknowns to be estimated. That is, the segmentation task is formulated as

$$
\begin{aligned}
\arg \max _{x_{s}, x_{s}^{(f)}} p\left(y_{s} \mid x_{s}\right) p\left(y_{s}^{(f)} \mid x_{s}^{(f)}\right) & \\
& \times P\left(x_{s}^{(f)} \mid x_{s}, \mathbf{x}_{\eta_{s}}\right) P\left(x_{s} \mid x_{s}^{(f)}, \mathbf{x}_{\eta_{s}}^{(f)}, \mathbf{x}_{\eta_{s}}\right)
\end{aligned}
$$

for each site $s$.

Equation (7) is the proposed objective function for the segmentation task. Unlike the MLL-based approaches, it uses two different context models for $P\left(x_{s} \mid x_{s}^{(f)}=0, \mathbf{x}_{\eta_{s}}^{(f)}, \mathbf{x}_{\eta_{s}}\right)$ and $P\left(x_{s} \mid x_{s}^{(f)}=1, \mathbf{x}_{\eta_{s}}^{(f)}, \mathbf{x}_{\eta_{s}}\right)$, respectively, as shown later in Section III-B. Of the two models, one favors large homogeneous regions and the other favors narrow elongated filaments. In (7), the two terms $p\left(y_{s}^{(f)} \mid x_{s}^{(f)}\right)$ and $P\left(x_{s}^{(f)} \mid x_{s}, \mathbf{x}_{\eta_{s}}\right)$ form the posterior as the following:

$$
P\left(x_{s}^{(f)} \mid y_{s}^{(f)}, x_{s}, \mathbf{x}_{\eta_{s}}\right) \propto p\left(y_{s}^{(f)} \mid x_{s}^{(f)}\right) P\left(x_{s}^{(f)} \mid x_{s}, \mathbf{x}_{\eta_{s}}\right) .
$$

Therefore, based on the evidence and the context of class labels, the existence of filaments is inferred and determines in the optimization whether the current site should use the model favoring homogeneous regions or switch to the model favoring filaments.

\section{A. Feature Model}

The feature models in (7) include those for $p\left(y_{s} \mid x_{s}\right)$ and $p\left(y_{s}^{(f)} \mid x_{s}^{(f)}\right)$. For $y_{s}$, which is gray level, there is no necessary difference in the modeling of $p\left(y_{s} \mid x_{s}\right)$ than in the traditional approaches [18]. A Gaussian distribution is assumed here.

For the filament strength $y_{s}^{(f)}$, the definition is based on Haralick's work on ridge detection [21]. In [21], the process first finds for each site a direction having greatest magnitude of second directional derivative, and then checks in that direction whether or not a zero crossing of first directional derivative has occurred. Those zero crossing sites correspond to the ridge peaks or valley bottoms, and hence the ridge (valley) sites can be located. In this paper, if a site $s$ is a zero crossing site the filament strength $y_{s}^{(f)}$ is defined as $\left|y_{s}^{\prime \prime}\left(\theta_{s}\right)\right|$, where $y_{s}^{\prime \prime}\left(\theta_{s}\right)$ is the second derivative in the main principle curvature direction $\theta_{s}$. In the model, $p\left(y_{s}^{(f)} \mid x_{s}^{(f)}\right)$ is assumed to be Gaussian. ${ }^{1}$ To reduce the influence of noise, a Gaussian smoothing is performed on the original SAR image before computing the derivatives.

\section{B. Context Model}

The model for $P\left(x_{s} \mid x_{s}^{(f)}, \mathbf{x}_{\eta_{s}}^{(f)}, \mathbf{x}_{\eta_{s}}\right)$ consists of two different components for filament and nonfilament labels.

1) Current site is nonfilament $\left(x_{s}^{(f)}=0\right)$. The MLL model in (3) is used, which favors the class label dominant in the neighborhood. The equation is presented again here as follows:

$$
V_{c}\left(x_{s}, x_{t}, x_{s}^{(f)}=0, x_{t}^{(f)}\right)= \begin{cases}\beta, & \text { if } x_{t} \neq x_{s} \\ 0, & \text { otherwise. }\end{cases}
$$

2) Current site is filament $\left(x_{s}^{(f)}=1\right)$. The class label of current site should be the same as that of filament neighbors and different from that of nonfilament neighbors. The clique energy is hence defined as

$$
\begin{aligned}
& V_{c}\left(x_{s}, x_{t}, x_{s}^{(f)}=1, x_{t}^{(f)}\right) \\
& \quad= \begin{cases}a \beta, & \text { if } x_{t}^{(f)}=1 \text { and } x_{t} \neq x_{s} \\
a \beta, & \text { if } x_{t}^{(f)}=0 \text { and } B_{s}\left(x_{t}, x_{s}\right)=\text { true } \\
0, & \text { otherwise. }\end{cases}
\end{aligned}
$$

Here, $\beta$ is a positive constant as in (3), and $a$ is a nonnegative real number. Also

$$
B_{s}\left(x_{t}, x_{s}\right)=\left\{\mu_{x_{s}} \leq \mu_{x_{t}}, y_{s}^{\prime \prime}\left(\theta_{s}\right)<0\right\} \text { or }\left\{\mu_{x_{s}} \geq \mu_{x_{t}}, y_{s}^{\prime \prime}\left(\theta_{s}\right)>0\right\}
$$

where $\mu_{x}$ is the mean gray level of class $x$. In (10), the formula penalizes two situations. In the first situation, $x_{t}$ and $x_{s}$ both belong to a filament but have different class labels. This is unlikely and should have a smaller prior corresponding to a higher clique energy. In the second situation, $B_{s}\left(x_{t}, x_{s}\right)=$ true corresponds to two possibilities. For the first, $y_{s}^{\prime \prime}\left(\theta_{s}\right)<0$ means that the current site $s$ belongs to a ridge and thus should be brighter than neighboring nonfilament sites, while $\mu_{x_{s}} \leq$ $\mu_{x_{t}}$ gives a contradictory observation. This is again not a desirable configuration and should be penalized. For the other possibility of $B_{s}\left(x_{t}, x_{s}\right)=$ true, a similar contradiction exists between $y_{s}^{\prime \prime}\left(\theta_{s}\right)>0$ (indicating a valley) and $\mu_{x_{s}} \geq \mu_{x_{t}}$ (indicating a nonvalley configuration) and is also penalized.

With $V_{c}\left(x_{s}, x_{t}, x_{s}^{(f)}, x_{t}^{(f)}\right)$ defined, $P\left(x_{s} \mid x_{s}^{(f)}, \mathbf{x}_{\eta_{s}}^{(f)}, \mathbf{x}_{\eta_{s}}\right)$ can be computed as $\exp \left\{-\sum_{t \in \eta_{s}} V_{c}\left(x_{s}, x_{t}, x_{s}^{(f)}, x_{t}^{(f)}\right)\right\} / Z$, where $Z=\sum_{x_{s}} \exp \left\{-\sum_{t \in \eta_{s}} V_{c}\left(x_{s}, x_{t}, x_{s}^{(f)}, x_{t}^{(f)}\right)\right\}$ is a normalizing factor computed by summation of the numerator over the configuration space of $x_{s}$.

\footnotetext{
${ }^{1}$ For zero crossing sites that are nonfilament, $y_{s}^{\prime \prime}\left(\theta_{s}\right)$ is mostly caused by noise and is zero mean. Therefore, $p\left(y_{s}^{(f)} \mid x_{s}^{(f)}=0\right)$ is in fact assumed to be half-sided Gaussian.
} 


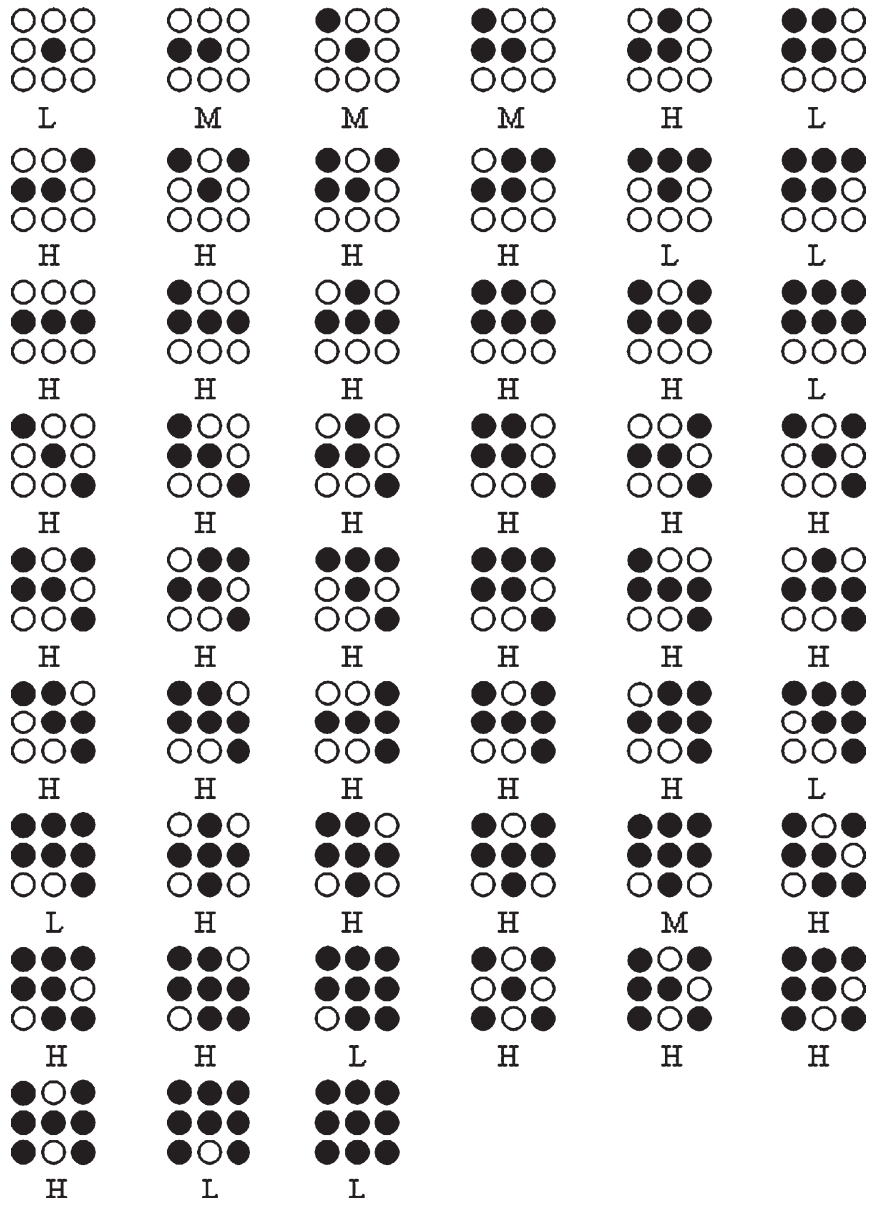

Fig. 2. Initial values of $P\left(x_{s}^{(f)}=1 \mid x_{s}, \mathbf{x}_{\eta_{s}}\right)$, the conditional probabilities of site $s$ being a filament given the class labels of itself and its neighbors. The central site is always black. Neighboring sites that have the same class label as the central one (i.e., $x_{t}=x_{s}, t \in \eta_{s}$ ) are black as well; otherwise, they are white.

$P\left(x_{s}^{(f)} \mid x_{s}, \mathbf{x}_{\eta_{s}}\right)$ is modeled as in Fig. 2. In the figure, the central site is always black. Neighboring sites that have the same class label as the central one (i.e., $x_{t}=x_{s}, t \in \eta_{s}$ ) are black as well; ${ }^{2}$ otherwise, they are white. $P\left(x_{s}^{(f)}=1 \mid x_{s}, \mathbf{x}_{\eta_{s}}\right)$ should be set to a large value if the site has limited number of black neighbors in separate directions, and vice versa. Fig. 2 lists some configurations, and the rest can be obtained by symmetry. In the figure, $P\left(x_{s}^{(f)}=1 \mid x_{s}, \mathbf{x}_{\eta_{s}}\right)$ has three different possible values of $L, M$, and $H$, which denote low, medium, and high probabilities, respectively. The first three patterns listed in Fig. 2 are the three most occurring ones. Their probability values are crucial. For the first configuration in Fig. 2, $P\left(x_{s}^{(f)}=1 \mid x_{s}, \mathbf{x}_{\eta_{s}}\right)$ should be as close to zero as possible to select the nonfilament model to suppress noisy configurations, but on the other hand should not be too small so that the filament model has the possibility to be used to link broken filaments. Considering the small population of broken filament sites compared to that of noise, the value 0.001 is chosen. For the next two configurations in Fig. 2, $P\left(x_{s}^{(f)}=1 \mid x_{s}, \mathbf{x}_{\eta_{s}}\right)$ is set to be 0.5 as the central site can equally be either noise or the end of a filament if there is no other information favoring one over the other. Therefore, we

\footnotetext{
${ }^{2}$ The eight-neighbor system is used in this paper.
}

set $L$ to be $0.001, M$ to be 0.5 , and $H$ to be 0.9 . Such values are only used initially. They are further refined by an expectationmaximization (EM) technique during the optimization process, as shown later in Section III-C. In fact, the initial values of all other configurations except the first three have little effect on the final result and can even be assigned random numbers in $\left[\begin{array}{ll}0 & 1\end{array}\right]$.

\section{Parameter Estimation}

Parameters to be estimated include: the Gaussian parameters for $p\left(y_{s} \mid x_{s}\right)$, the Gaussian parameters for $p\left(y_{s}^{(f)} \mid x_{s}^{(f)}\right)$, the conditional probabilities of $P\left(x_{s}^{(f)} \mid x_{s}, \mathbf{x}_{\eta_{s}}\right)$, and $a$ and $\beta$ of the context model.

1) $P\left(y_{s} \mid x_{s}\right)$ : The assumption of $p\left(y_{s} \mid x_{s}\right)$ being Gaussian means that the observation $y_{s}$ is a mixture of $n$ Gaussian functions, where $n$ is the number of classes. The EM algorithm [22] is typically used to determine the parameters for Gaussian mixture.

Let $P_{i}, \mu_{i}$, and $\sigma_{i}^{2}$ denote the prior, mean and variance for class $i$, respectively, and $w_{s i}$ represents the probability of site $s$ belonging to class $i$ given the observation data and current estimate of all parameters. ${ }^{3}$ The EM algorithm iterates through computing the E step

$$
w_{s i}= \begin{cases}1, & \text { if } \forall j, p\left(y_{s} \mid \mu_{i}, \sigma_{i}\right) P_{i} \geq p\left(y_{s} \mid \mu_{j}, \sigma_{j}\right) P_{j} \\ 0, & \text { otherwise }\end{cases}
$$

and the M step

$$
\begin{aligned}
\mu_{i} & =\frac{\sum_{s} w_{s i} y_{s}}{\sum_{s} w_{s i}} \\
\sigma_{i}^{2} & =\frac{\sum_{s} w_{s i}\left(y_{s}-\mu_{i}\right)^{2}}{\sum_{s} w_{s i}} \\
P_{i} & =\frac{\sum_{s} w_{s i}}{\sum_{i} \sum_{s} w_{s i}} .
\end{aligned}
$$

2) $P\left(y_{s}^{(f)} \mid x_{s}^{(f)}\right)$ and $p\left(x_{s}^{(f)} \mid x_{s}, \mathbf{x}_{\eta_{s}}\right)$ : Similar to $p\left(y_{s} \mid x_{s}\right)$, this is also a Gaussian mixture problem with respect to the filament strength feature $y_{s}^{(f)}$. Again, the EM technique is used to estimate the parameters. Let $\mu_{i}^{(f)}$ and $\sigma_{i}^{(f) 2}$ denote the mean and variance of $y_{s}^{(f)}$ for configuration $i$. The E step is

$$
w_{s i}=\frac{p\left(y_{s}^{(f)} \mid \mu_{i}^{(f)}, \sigma_{i}^{(f)}\right) P\left(i \mid x_{s}, \mathbf{x}_{\eta_{s}}\right)}{\sum_{i} p\left(y_{s}^{(f)} \mid \mu_{i}^{(f)}, \sigma_{i}^{(f)}\right) P\left(i \mid x_{s}, \mathbf{x}_{\eta_{s}}\right)}
$$

and the $\mathrm{M}$ step is

$$
\mu_{i}^{(f)}=\frac{\sum_{s} w_{s i} y_{s}^{(f)}}{\sum_{s} w_{s i}}
$$

\footnotetext{
${ }^{3}$ Although $w_{s i}$ can be any number in [0 1], here only the two values of 0 and 1 are allowed based on the provisional segmentation.
} 


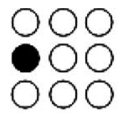

(a)

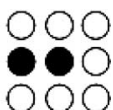

(b)
Fig. 3. (a) Center site belongs to the background. (b) The center site is on the tail of a filament.

$$
\begin{aligned}
\sigma_{i}^{(f) 2} & =\frac{\sum_{s} w_{s i}\left(y_{s}^{(f)}-\mu_{i}^{(f)}\right)^{2}}{\sum_{s} w_{s i}} \\
P(i \mid u, \mathbf{v}) & =\frac{\sum_{s \in S_{u \mathbf{v}}} w_{s i}}{\sum_{i} \sum_{s \in S_{u \mathbf{v}}} w_{s i}}
\end{aligned}
$$

where in (18) $S_{u \mathbf{v}}=\left\{s \mid x_{s}=u, \mathbf{x}_{\eta_{s}}=\mathbf{v}\right\}$. Considering that a half-sided Gaussian function is involved in the mixture, $\mu_{0}^{(f)}$ is simply set to zero at each iteration in the above EM procedures. Here, the EM steps iterate along with the segmentation. At each iteration, the class labels $\mathbf{x}$ are updated, and an E step followed by an $\mathrm{M}$ step is performed for refining the parameters. Equation (18) computes the prior conditioned on the class label configurations in the defined neighborhood, and thus $P\left(x_{s}^{(f)} \mid x_{s}, \mathbf{x}_{\eta_{s}}\right)$ is obtained.

3) $\beta$ and a: Typically, the MLL parameter $\beta$ is assigned a value based on experimentation. For example, Rignot and Chellappa [23] reported that [1.0-1.6] is the best range for $\beta$ in their polarimetric SAR image segmentation. Yue [24] believes that $\beta$ could be set between 1 and 3 based on experiments on SAR sea ice imagery. The difficulty of the problem is due to the intractability of the normalizing constant of the distribution, known as the partition function, that makes direct maximumlikelihood (ML) estimation of $\beta$ impossible. There are some efforts that try to maximize an approximation of the likelihood [20], [25] or use a Monte Carlo scheme [26] to avoid the computation of the partition function. In this paper, $\beta$ is simply chosen to be 2 . Some smaller values have been tested, but noise appears at such levels.

The role of $a$ is to put more weight on class label configurations that are consistent with current estimate of filament flag configurations, and suppress those that are not. Larger values of $a$ suggests greater power for detecting and preserving filaments but may also weaken the ability of the model in suppressing noisy results. For choosing the appropriate $a$, consider a special cases in Fig. 3.

In this example, only one of the neighbors belongs to class A (represented by black) and all other neighbors belong to class B (represented by white). The filament flag of the black neighbor is 1. This situation will generally happen near the two end points of filaments. Suppose the filament strength and the class label context tell us nothing about whether the center site is on the tail of a filament or not, i.e., $P\left(x_{s}^{(f)}=0 \mid y_{s}^{(f)}, x_{s}, \mathbf{x}_{\eta_{s}}\right)=$ $P\left(x_{s}^{(f)}=1 \mid y_{s}^{(f)}, x_{s}, \mathbf{x}_{\eta_{s}}\right)=0.5$, then the classification of the site should not be influenced by the context model but only depends on its gray level. Since $P\left(x_{s}^{(f)} \mid y_{s}^{(f)}, x_{s}, \mathbf{x}_{\eta_{s}}\right)$ is now a constant and based on (8), (7) becomes

$$
\arg \max _{x_{s}, x_{s}^{(f)}} p\left(y_{s} \mid x_{s}\right) P\left(x_{s} \mid x_{s}^{(f)}, \mathbf{x}_{\eta_{s}}^{(f)}, \mathbf{x}_{\eta_{s}}\right) .
$$

Any bias imposed by the context model of $P\left(x_{s} \mid x_{s}^{(f)}, \mathbf{x}_{\eta_{s}}^{(f)}\right.$, $\left.\mathbf{x}_{\eta_{s}}\right)$ is not desirable. Therefore, while the nonfilament model in (9) favors the configuration of Fig. 3(a) and the filament model in (10) favors that of Fig. 3(b), their influences should be equal in the amount so that the two configurations are equally probable. By the nonfilament model, the context model energy of Fig. 3(a) is $\beta$, and that of Fig. 3(b) is $7 \beta$. By the filament model, the context model energy of Fig. 3(a) is $8 a \beta$, and that of Fig. 3(b) is 0 . Therefore, the context model energy difference between the two configurations is $-6 \beta$ using the nonfilament model, and $8 a \beta$ using the filament model. Thus, $a=0.75$.

In practice, depending on the image, we often want the overall process to weight on one or the other of the two models to emphasize on suppressing noise or detecting filaments. $a$ is thus not necessary to be 0.75 exactly, but can be smaller or larger depending on the image characteristics.

\section{Variable Weighting}

In this paper, the simulated annealing (SA) [27] is adopted as the optimization technique. During the iterative optimization process, feature model parameters are gradually refined based on (11)-(18). However, the influence of context model over a beginning random class label configuration can possibly be quite misleading for such a parameter estimation, producing an unpredictable result. Therefore, following [11], a variable weighting parameter (V-MLL) is introduced to adjust adaptively the relative importance of the feature model over the context model during the segmentation process. The objective function of (7) is changed to

$$
\begin{aligned}
\arg \max _{x_{s}, x_{s}^{(f)}} & \left(p\left(y_{s} \mid x_{s}\right) p\left(y_{s}^{(f)} \mid x_{s}^{(f)}\right)\right)^{\alpha} \\
& P\left(x_{s}^{(f)} \mid x_{s}, \mathbf{x}_{\eta_{s}}\right) P\left(x_{s} \mid x_{s}^{(f)}, \mathbf{x}_{\eta_{s}}^{(f)}, \mathbf{x}_{\eta_{s}}\right) .
\end{aligned}
$$

At first, $\alpha$ is set to a large value so that the feature model dominates and the spatial relationship information can be ignored. As the segmentation process iterates, $\alpha$ is decreased to let the context model play a more important role.

\section{EXPERIMENTS AND DISCUSSIONS}

\section{A. Experiment Setup}

For the ridge feature extraction, a Gaussian filter of variance 12 is used in computing the main principle curvature direction. With the direction obtained, the original image is then smoothed by a Gaussian filter of variance 3 along that direction and by a filter of variance 12 normal to the direction. The ridge feature is then computed based on the smoothed image.

Four methods are compared: clustering based on the Gaussian mixture model (GMM), the MLL model with constant weighting (C-MLL) [18], the MLL model with V-MLL [11], and the proposed FPM. The SA technique [27] is used for all the approaches except GMM, and the following annealing schedule 
is applied:

$$
T(k)=T(0) * 0.95^{k}
$$

where $k$ is the iteration number. Initial temperature $T(0)$ is always set to be 1 . For the V-MLL $\alpha$ in V-MLL and FPM, the same scheme as follows is used [11]:

$$
\alpha(k)=80 * 0.9^{k}+1 .
$$

The parameter $a$ of the FPM is adjusted around 0.75 for different tested images. It has been found that the segmentation results are similar for a moderate range of values of $a$ around 0.75 . Of the two kinds of images tested later in the experiments (one synthetic and the other SAR sea ice), the synthetic one is with heavier noise and a smaller value of 0.6 is applied. For the SAR sea ice images, we simply choose $a=0.9$ based on experiments.

For quantitative evaluations of the results, two metrics are used. The first is the overall accuracy (OA) which, as typically defined, is the ratio of the number of correctly labeled pixels over the total number of image sites. For situations where the boundary and filament sites occupy only a small fraction of the image, a second metric named boundary accuracy (BA) is used, which is the ratio of the number of correctly labeled pixels in the boundary region over the total number of sites in the boundary region. Here, the boundary region is defined based on the ground truth image to be the set of sites whose minimum Euclidean distance to boundary sites (i.e., those that have at least a neighbor belonging to a different class) is no more than 2.

\section{B. Segmentation of Synthetic Image}

Fig. 4(a) is a synthetic "star" shape $523 \times 501$ image corrupted by additive Gaussian noise, and Fig. 4(b) is the truth. The image has two classes with mean gray levels of 128 and 178, respectively, and the same variance of 650.25. Fig. 4(c)-(f) show, respectively, the results of segmentation using the GMM, C-MLL, V-MLL, and the proposed model FPM. The result by the GMM is unacceptable, while the other three approaches are much more effective. This shows the importance of spatial context to image segmentation. The C-MLL result in Fig. 4(d) has noticeable errors inconsistent with the "star" shape, and lags in classification accuracy to V-MLL in Fig. 4(e) and FPM in Fig. 4(f). Compared to the C-MLL and V-MLL approaches, the proposed FPM model generates visually improved results with noticeably better defined shapes and longer tails. Although the OA in Fig. 4(f) is only marginally better than in Fig. 4(e), this is due to the fact that the filaments only occupy a very small population of the image sites. By comparing the BA percentage, the FPM is shown to have improved over the V-MLL by $4 \%$. The advantage of the proposed model over the C-MLL and V-MLL models in preserving filaments is obvious in the difference images shown in Fig. 4(g)-(i), where in Fig. 4(i) erroneously labeled sites (white sites) are significantly fewer in the tails. Fig.4 (j) gives the filament map obtained simultaneously with the segmentation. The black sites in this map indicates the locations where the filament model is used instead of the nonfilament MLL model. Validation of the Gaussian model for the filament strength is investigated in Fig. 5. In the

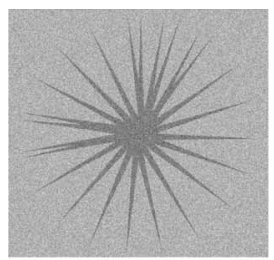

(a)

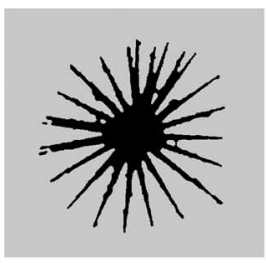

(d)

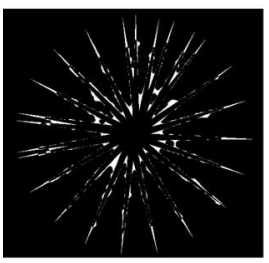

(g)

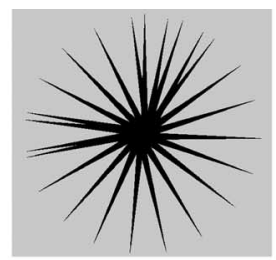

(b)

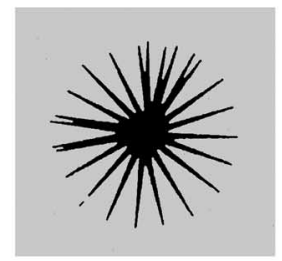

(e)

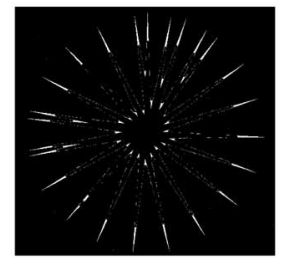

(h)

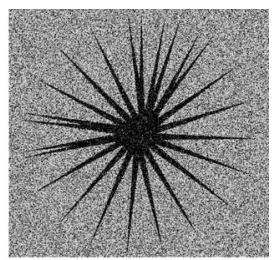

(c)

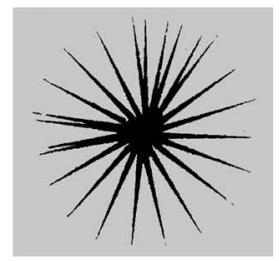

(f)

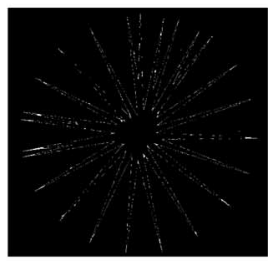

(i)

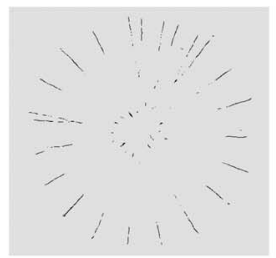

(i)

Fig. 4. (a) Synthetic noisy "star" image; (b) Truth; Results: (c) GMM (OA: $77.8 \%$, BA: 78.7\%); (d) C-MLL (OA: 96.6\%, BA: 84.0\%); (e) V-MLL (OA: $98.1 \%$, BA: $90.8 \%)$; (f) FPM $(a=0.6$, OA: $99.0 \%$, BA: $94.8 \%)$; (g) Difference between C-MLL result and truth; (h) Difference between V-MLL result and truth; (i) Difference between FPM result and truth; (j) The filament map.

graph, the distribution of the normalized $y_{s}^{(f)}$ is highly peaked at the zero point and have a long tail toward 1. The GMM gives a close approximation for the distribution.

\section{Segmentation of SAR Sea-Ice Imagery}

Fig. 6(a) is an image of Gulf of St. Lawrence acquired by RADARSAT ScanSAR (C-band, HH, 100-m pixel spacing) on February 19, 1997. This image has an bimodal histogram, suggesting that the features of ice and water are quite separable. However, existence of noise has led to a "spotty" result using the GMM Fig. 6(b). In Fig. 6(c) and (d) incorporation of spatial context by MLL gives an improved result, but at the same time details of filaments are lost. The proposed model in Fig. 6(e) performs the best. More cracks and longer tails can clearly be seen, as indicated by the details shown in Fig. 6(f). Using manual segmentations as the truth, the overall accuracies and boundary accuracies are computed from the eight subregions in Fig. 6(f), together as a whole, for quantitative evaluations. These accuracies are reported in the caption for Fig. 6. The FPM is shown to have significantly better performance, with a BA $8.9 \%$ higher and an OA $3.1 \%$ higher than the second best approach (V-MLL). 


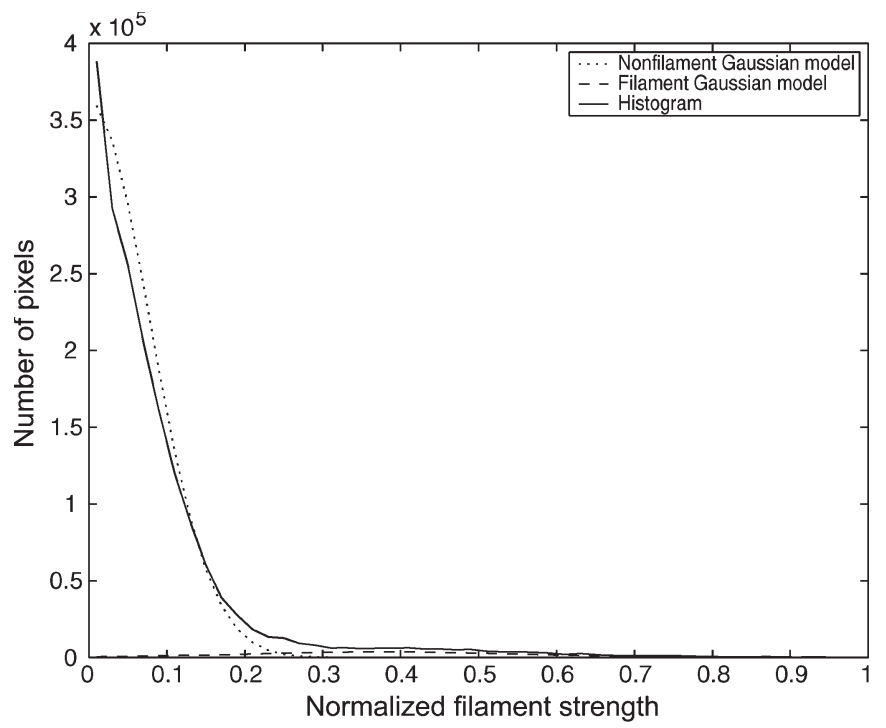

Fig. 5. Obtained Gaussian models for filament strength for Fig. 4.

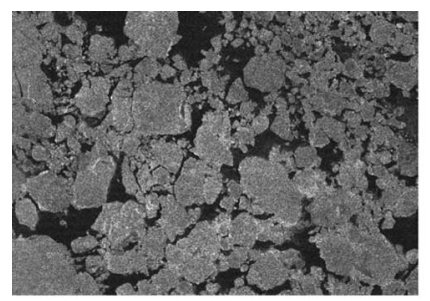

(a)

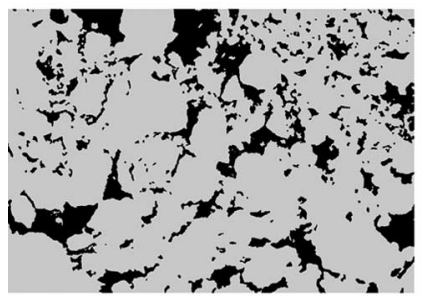

(c)

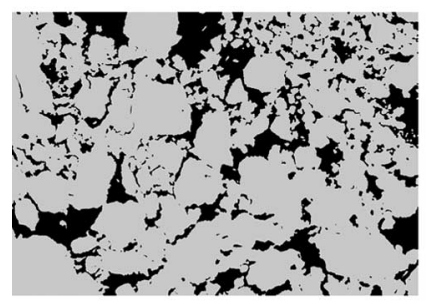

(e)

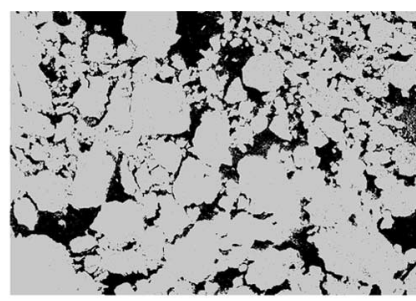

(b)

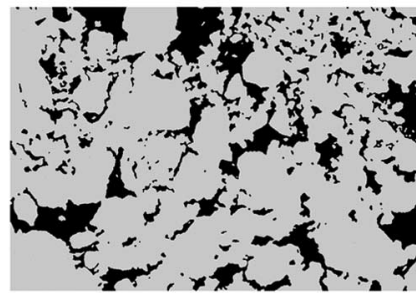

(d)

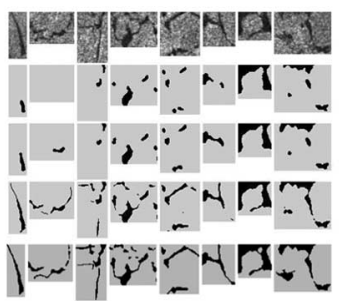

(f)
Fig. 6. (a) SAR image of Gulf of St. Lawrence on February 19, 1997 acquired by RADARSAT ScanSAR (C-band, HH, 100-m pixel spacing); Results: (b) GMM; (c) C-MLL; (d) V-MLL; (e) FPM ( $a=0.9$ ); (f) Detail comparison. First row: original; second row: C-MLL (OA: 92.4\%, BA: 78.0\%); third row: V-MLL (OA: 93.5\%, BA: 81.4\%); fourth row: FPM (OA: 96.6\%, BA: 90.3\%); fifth row: manual segmentation (truth).

Fig. 7(a) is another RADARSAT image of the Gulf of St. Lawrence acquired on February 20, 1998. This image is well understood as it is used for training purposes at the CIS. Excluding the land in the bottom, the image has three classes. The dark region surrounding the land is water. The bright region from the top left to the center of the image is gray ice, and the rest is gray-white ice. This image has many long

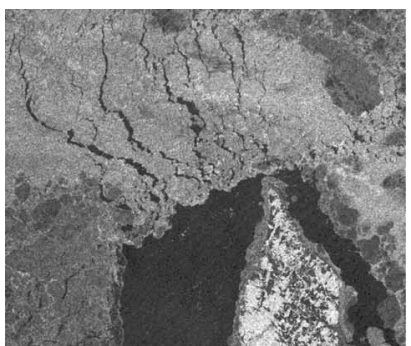

(a)

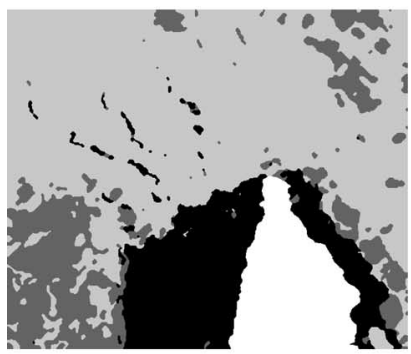

(c)

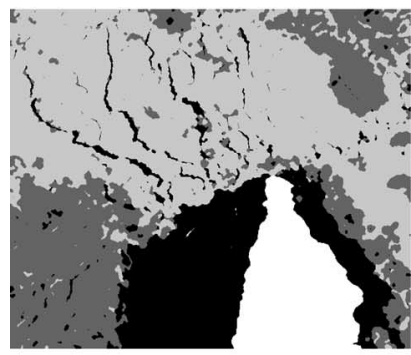

(e)

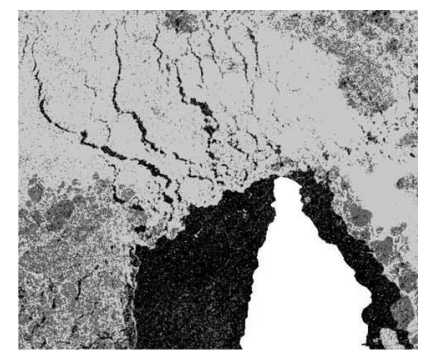

(b)

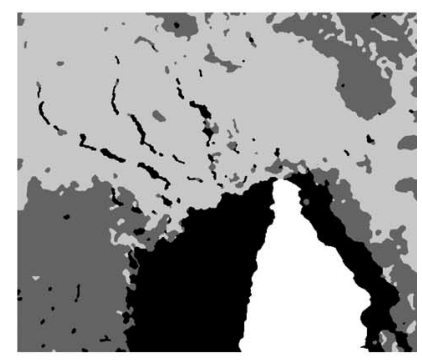

(d)

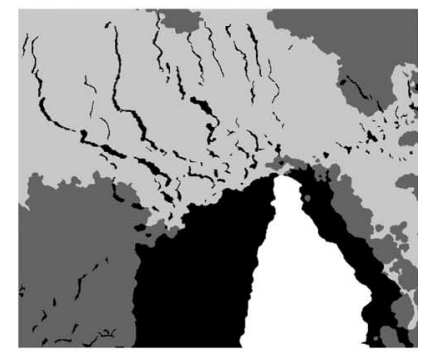

(f)
Fig. 7. (a) SAR image of Gulf of St. Lawrence on February 20, 1998 acquired by RADARSAT ScanSAR (C-band, HH, 100-m pixel spacing); Results: (b) GMM (OA: 62.4\%, BA: 47.2\%); (c) C-MLL (OA: 83.0\%, BA: 61.8\%); (d) V-MLL (OA: 91.9\%, BA: 71.7\%); (e) FPM $(a=0.9$, OA: $92.0 \%$, BA: $75.3 \%$ ); (f) Manual segmentation (truth).

narrow leads clearly visible in the gray ice region, and thus is suitable for testing the proposed model. In Fig. 7(b), the GMM model is able to identify the lead structure, but the regions have tremendous errors. This motivates the proposed method that can simultaneously capture the lead structure and segment regions accurately. Compared to the C-MLL result in Fig. 7(c) and V-MLL result in Fig. 7(d), the FPM approach in Fig. 7(e) has preserved more narrow elongated segments. The FPM has also connected some segments that are broken in Fig. 7(c) and (d), forming long narrow leads. Quantitative evaluations based on a manual segmentation is performed, and also shows the advantages of the proposed FPM method. The FPM has a BA 3.6\% higher than the method with the second highest accuracy (V-MLL).

\section{Summary AND Future Work}

We have proposed a filament-preserving model capable of preserving narrow linear features without losing the strength in suppressing noisy configurations. Experiments have shown the promise of this method in application of SAR sea-ice analysis.

Improvement of this paper may be achieved by integrating texture features and refining the gray level feature model with Gamma distribution. It has also been noticed that while 
our method tries to alleviate the undersegmentation problem specifically for filaments, it may suffer the oversegmentation problems for some SAR sea ice imagery. It is well known that multiscale-based methods can improve significantly the oversegmentation problem, and therefore another future work would be extending this model in a multiscale manner.

\section{ACKNOWLEDGMENT}

RADARSAT images are copyright of the Canadian Space Agency (CSA).

\section{REFERENCES}

[1] W. M. O., Dec. 17, 2005. [Online]. Available: http://www.wmo.ch/indexen.html

[2] A. Baraldi and F. Parmiggiani, "An investigation of the textural characteristics associated with gray level cooccurrence matrix statistical parameters," IEEE Trans. Geosci. Remote Sens., vol. 33, no. 2, pp. 293-304, Mar. 1995.

[3] D. G. Barber and E. F. LeDrew, "SAR sea ice discrimination using texture statistics: A multivariate approach," Photogramm. Eng. Remote Sens., vol. 57, no. 4, pp. 385-395, 1991.

[4] M. E. Shokr, "Evaluation of second-order texture parameters for sea ice classification from radar images," J. Geophys. Res., vol. 96, no. C6, pp. 10625-10640, 1991.

[5] L. K. Soh and C. Tsatsoulis, "Texture analysis of SAR sea ice imagery using gray level co-occurrence matrices," IEEE Trans. Geosci. Remote Sens., vol. 37, no. 2, pp. 780-795, Mar. 1999.

[6] _ - "Unsupervised segmentation of ERS and RADARSAT sea ice images using multiresolution peak detection and aggregated population equalization," Int. J. Remote Sens., vol. 20, no. 15/16, pp. 3087-3109, 1999.

[7] R. Samadani, "A finite mixture algorithm for finding proportions in SAR images," IEEE Trans. Image Process., vol. 4, no. 8, pp. 1182-1186, Aug. 1995.

[8] D. A. Clausi and B. Yue, "Comparing co-occurrence probabilities and Markov random fields for texture analysis of SAR sea ice imagery," IEEE Trans. Geosci. Remote Sens., vol. 42, no. 1, pp. 215-228, Jan. 2004.

[9] L. K. Soh, C. Tsatsoulis, D. Gineris, and C. Bertoia, "ARKTOS: An intelligent system for SAR sea ice image classification," IEEE Trans. Geosci. Remote Sens., vol. 42, no. 1, pp. 229-248, Jan. 2004.

[10] J. A. Karvonen, "Baltic sea ice SAR segmentation and classification using modified pulse-coupled neural networks," IEEE Trans. Geosci. Remote Sens., vol. 42, no. 7, pp. 1566-1574, Jul. 2004.

[11] H. Deng and D. A. Clausi, "Unsupervised segmentation of synthetic aperture radar sea ice imagery using a novel Markov random field model," IEEE Trans. Geosci. Remote Sens., vol. 43, no. 3, pp. 528-538, Mar. 2005.

[12] J. F. Vesecky, M. P. Smith, and R. Samadani, "Extraction of lead and ridge characteristics from SAR images of sea ice," IEEE Trans. Geosci. Remote Sens., vol. 28, no. 4, pp. 740-744, Jul. 1990.

[13] A. Garzelli, "Classification of polarimetric SAR images using adaptive neighborhood structures," Int. J. Remote Sens., vol. 20, no. 8, pp. 1669$1675,1999$.

[14] P. C. Smits and S. G. Dellepiane, "Synthetic aperture radar image segmentation by a detail preserving Markov random field approach," IEEE Trans. Geosci. Remote Sens., vol. 35, no. 4, pp. 844-857, Jul. 1997.

[15] J. Besag, "Spatial interaction and the statistical analysis of lattice systems," J. R. Stat. Soc., Ser. B, vol. 36, no. 2, pp. 192-236, 1974.

[16] H. Derin and H. Elliott, "Modeling and segmentation of noisy and textured images using Gibbs random fields," IEEE Trans. Pattern Anal. Mach. Intell., vol. PAMI-9, no. 1, pp. 39-55, Jan. 1987.

[17] S. Z. Li, Markov Random Field Modeling in Image Analysis. New York: Springer-Verlag, 2001.
[18] C. S. Won and H. Derin, "Unsupervised segmentation of noisy and textured images using Markov random fields," Comput. Vis. Graph. Image Process., Graph. Models Image Process., vol. 54, no. 4, pp. 308-328, 1992.

[19] R. O. Duda, P. E. Hart, and D. G. Stork, Pattern Classification. Hoboken, NJ: Wiley, 2001.

[20] J. Besag, "On the statistical analysis of dirty pictures," J. R. Stat. Soc., Ser. B, vol. 48, no. 3, pp. 259-302, 1986.

[21] R. M. Haralick, "Ridges and valleys on digital images," Comput. Vis. Graph. Image Process., vol. 22, no. 1, pp. 28-38, 1983.

[22] A. P. Dempster, N. M. Laird, and D. B. Rubin, "Maximum likelihood from incomplete data via the EM algorithm," J. R. Stat. Soc., Ser. B, vol. 39, no. 1 , pp. 1-38, 1977.

[23] E. Rignot and R. Chellappa, "Segmentation of polarimetric synthetic aperture radar data," IEEE Trans. Image Process., vol. 1, no. 3, pp. 281300, Jul. 1992.

[24] B. Yue, "SAR sea ice recognition using texture methods," M.S. thesis, Dept. Syst. Des. Eng., Univ. Waterloo, Waterloo, ON, Canada, 2001.

[25] S. Lakshmanan and H. Derin, "Simultaneous parameter estimation and segmentation of Gibbs random field using simulated annealing," IEEE Trans. Pattern Anal. Mach. Intell., vol. 11, no. 8, pp. 799-813, Aug. 1989.

[26] X. Descombes, R. D. Morris, J. Zerubia, and M. Berthod, "Estimation of Markov random field prior parameters using Markov chain Monte Carlo maximum likelihood," IEEE Trans. Image Process., vol. 8, no. 7, pp. 954-963, Jul. 1999.

[27] S. Geman and D. Geman, "Stochastic relaxation, Gibbs distributions, and the Bayesian restoration of images," IEEE Trans. Pattern Anal. Mach. Intell., vol. PAMI-6, no. 6, pp. 721-741, Nov. 1984.

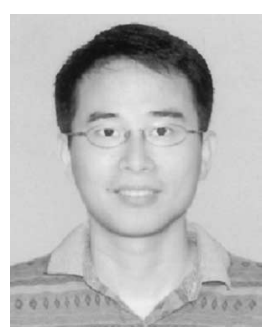

Qiyao Yu received the B.A.Sc. degree from Tsinghua University, Beijing, China, in 1997, the M.A.Sc. degree from the Memorial University of Newfoundland, St. John's, NL, Canada, in 2002, and the Ph.D. degree in systems design engineering from the University of Waterloo, Waterloo, ON, Canada, in 2006.

His research interest is in image processing and remote sensing.

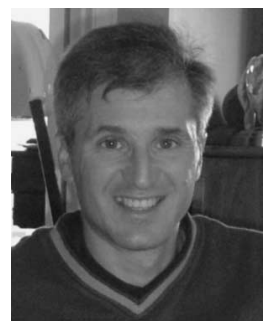

David A. Clausi (S'93-M'96-SM'03) received the B.A.Sc., M.A.Sc., and Ph.D. degrees from the University of Waterloo, Waterloo, ON, Canada, in 1990, 1992, and 1996, respectively, all in systems design engineering.

After completing his doctorate, he worked in the medical imaging field at Mitra Imaging, Inc., Waterloo. He started his academic career in 1997 as an Assistant Professor in geomatics engineering with the University of Calgary, AB, Canada. In 1999, he returned to his alma mater and was awarded tenure and promotion to Associate Professor in 2003. He is an active Interdisciplinary and Multidisciplinary Researcher. He has an extensive publication record, publishing refereed journal and conference papers in the diverse fields of remote sensing, computer vision, algorithm design, and biomechanics. His primary research interest is the automated interpretation of SAR sea-ice imagery, in support of operational activities of the Canadian Ice Service. The research results have led to successfully commercial implementations.

Dr. Clausi has received numerous scholarships, conference paper awards, and two Teaching Excellence Awards. 